De Boeck, S., Van Hertem, D. (2013). Coordination of Multiple HVDC Links in Power Systems during Alert and Emergency Situations. Proc. IEEE Powertech 2013, Grenoble, France, 16 - 20 June 2015, 6 pages.

Digital Object Identifier:

$\underline{10.1109 / P T C .2013 .6652278}$

URL:

http://ieeexplore.ieee.org/stamp/stamp.jsp?tp=\&arnumber=6652278

(C2013 IEEE. Personal use of this material is permitted. Permission from IEEE must be obtained for all other users, including reprinting/ republishing this material for advertising or promotional purposes, creating new collective works for resale or redistribution to servers or lists, or reuse of any copyrighted components of this work in other works. 


\section{Coordination of Multiple HVDC Links in Power Systems during Alert and Emergency Situations}

\author{
Steven De Boeck \\ Graduate Student Member, IEEE \\ Department of Electrical Engineering, ELECTA \\ KU Leuven, Leuven \\ Steven.deboeck@esat.kuleuven.be
}

\author{
Dirk Van Hertem \\ Senior Member, IEEE \\ Department of Electrical Engineering, ELECTA \\ KU Leuven, Leuven \\ Dirk.vanhertem@esat.kuleuven.be
}

\begin{abstract}
The increased share of distributed generation led to a higher loading of the transmission grid. TSOs have addressed the lack of investments in the grid due to the limited social acceptance of new transmission lines, through the installation of flow controllable devices. These devices allow to operate the power system closer to its limits. These devices can also be used to limit the impact of severe contingencies in the system. This paper addresses the effect of coordinated control of these devices on a small example case. By determining the HVDC set-points in a coordinated way, it is possible to extend the grid capabilities. The coordination of the power flow controlling devices can be done as a first measure in the security management procedures.
\end{abstract}

\section{INTRODUCTION}

The European policy goals on sustainability, security of supply and improved competiveness resulted in an increased share of renewables in generation, which in turn led to a higher loading of the transmission and distribution grids. These renewable generation units are located where wind and sun are readily available or financially beneficial. This is not necessarily where the load centers and available transmission infrastructures are located. Renewable energy sources are often variable and to a large extend unpredictable sources of electrical power, which are uncontrollable or not centrally dispatchable. As a result, the foreseen and even the actual operating point of the system is less certain than it was before. Also the liberalization of the European market and the transition to one single European electricity market led to a higher loading of connections between control zones. The traditional way of coping with higher loading of grids was the construction of extra overhead lines. This solution is no longer socially accepted. In turn these changes have led to the exploration of new ways of planning and operating of the transmission grids. [1] [2] [3] [4]

The consequence is that the transmission system is being operated closer to its limits and the risk for events that effect the power system security in a large area has considerably increased. Though TSOs have invested in technologies to control these changes in power flow and in technologies to increase the trade between different control zones, such as PSTs and HVDC point to point connections. The coordinated

The research leading to these results has received funding from the European Union Seventh Framework Programme under grant agreement no 283012 control of these devices has up till now only been considered during normal operation or during special protection schemes. A coordinated control mechanism for these devices during alert and emergency state is still lacking, certainly considering multiple zones. A consequence is that the part of the transmission capacity of these lines, that is reserved for security reasons is often based on experience.

The following shows how power flow controllable devices can help during alert and emergency condition when the grid is evolving towards an unsecure state. The objective is to minimize the impact of HILP contingencies (high impact low probability). The goal of the control methodology is to minimize cost, taking into account the costs related to the amount of load that needs to be shed to get the system back into a stable state. To reach this goal the set-points of the controllable devices will be adjusted. To quantify this, an estimation is made of the cost and the amount of energy not served. Subsequently some existing issues of implementing these solutions will be pointed out.

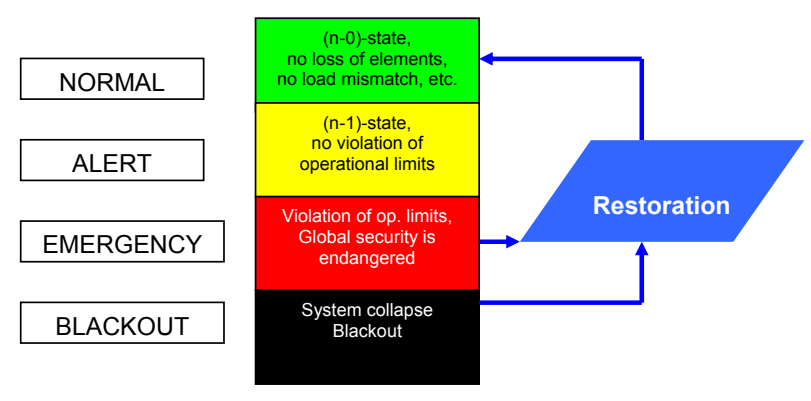

Fig. 1. The different grid states [5]

The second section elaborates on the alert and emergency state and how the secure spaces are defined. It also clarifies how the operation point moves within this space as a function of the grid controls. The next section describes the test system that has been used in the simulations. Subsequently a theoretic approach on how power controllable devices can contribute in extending the grid capabilities, is given. Section five describes the OPF model and the model used for the HVDC connections. Thereafter, the results of the simulation are discussed. The final section formulates the conclusions. 


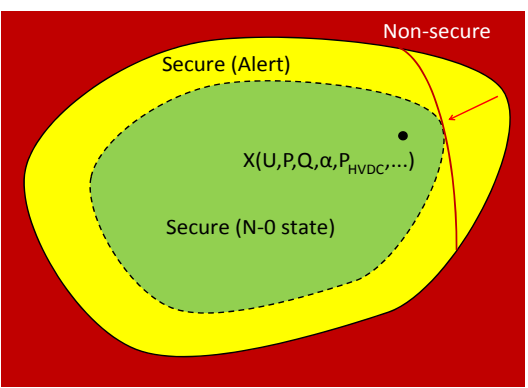

Fig. 2. The secure and unsecure space

\section{Controlling the Alert And EMERGEnCy StAte}

Policy 5 of the operation handbook of ENTSO-E gives the following description for alert and emergency situations [5].

- Alert: Risk for interconnected system operation, though the system operation is within acceptable limits. The TSO has uncertainties to come back to a normal state after one or more contingencies.

- Emergency: The system is in a deteriorated situation with a higher risk for neighbouring systems. Security principles are not fullfiled and the global security is endangered. There is no guarantee of total efficiency of remedies to limit propagation to neighbouring systems or to the whole ENTSO-E system.

In figure 1 and 2 , the alert state is colored yellow and the emergency state red.

When the system enters the alert or emergency state, the system is no longer $\mathrm{N}-1$ secure. The main concern of the TSO at this point is to maintain the system integrity and, while trying to achieve this, to maintain the supply to as many customers as possible. The first steps of the defense plan mainly consist of re-dispatching, starting up of plants to make sufficient generation available and removing some of the load (ex. stop pumping of pumping plants). If needed reactive power generation is also increased.

Figure 2 gives an abstract representation of the operating space of the system. The black dot represents the current operating point of the system $\mathrm{X}(\mathrm{U}, \mathrm{P}, \mathrm{Q}, \ldots)$. The location of this point depends on a number of variables, such as the active power injection and off take $\mathrm{P}$, the reactive power injections and off take $\mathrm{Q}$, the set points of the phase shifters $(\alpha)$ and the set points of the HVDC connections in the system $\left(P_{h v d c}\right.$ and $\left.Q_{h v d c}\right)$. The outer black line represents the security border of this system. This is the theoretical limit, that is determined by all the boundary conditions of the system. Such boundary conditions can be the thermal limits of the branches in the system or the transient and voltage stability limits. In theory, it should be possible to operate the system up to this border, without directly resulting into a loss of load or even a blackout. The space outside this region thus is unsecure. The space confined by the dashed line is the $\mathrm{N}-1$ secure space (or can be replaced by any other reliability criteria in use by the operator). During normal operation the operating point of the system should always remain within this space. These spaces should

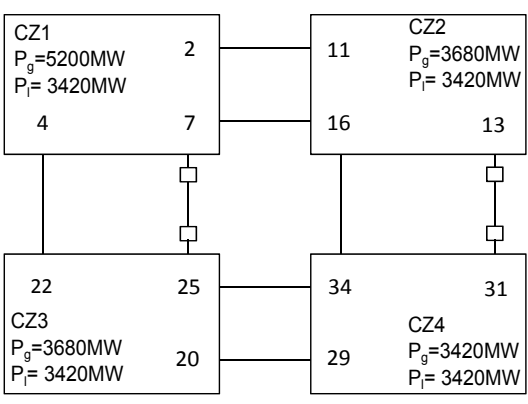

Fig. 3. Simplified presentation of the grid

not be considered to be static, as both the boundary condition and the operating point can change continuously. An example could be the tripping of a line, which causes a reduction of the secure space. This is shown by the arrow in figure 2. During the emergency state, some of the systems operating limits are violated. During the alert state, a contingency has occurred, but a violation of the operational limits has not yet taken place. The alert state results in an operating point between the dashed and full line. At this point, the system operator has a number of possibilities to bring the system to a more secure state. A first option is the re-dispatch of generation, which causes the operating to point to move away from the secure border to a more secure point. In a liberalized context this comes at a considerable cost. A second solution consists of topology changes (line switching), which can enlarge the secure space near the operating point and can increase the distance between this point and the secure border. The solution considered here, is changing the set points of the power flow controllable devices (phase shifting transformers and HVDC connections) in the system. The controllable devices are generally owned and operated by TSOs and might offer a relatively cheap control option. One of the advantages of the HVDC line is the fast response to a change in set point compared to the start-up of extra generation.

\section{DESCRIPTION OF THE TEST CASE}

The test case used to illustrate the effect of the power flow control during operation in emergency state of the power system, consists of four control zones (CZ) and is based on the cloverfield system in figure 4 [2]. The system consists of four symmetrical subsystems connected together. The sole difference between the subsystems is the cost of the generation. $\mathrm{CZ} 1$ has the cheapest generation, while $\mathrm{CZ} 4$ has the most expensive. CZ 2 and $C Z 3$ have equal and intermediate costs of generation. This result in a small net flow from $C Z 1$ to $C Z 4$. The CZ 1 and CZ 2 are interconnected by two AC lines: 2-11 and 7-16. Also the CZ 3 and $\mathrm{CZ} 4$ are interconnected by two AC lines: 20-29 and 25-34. In this system two HVDC lines are placed: one between bus 7 and 25 and the other between bus 13 and 31. There is also an AC connection between CZ1 and CZ2 (4-22) and one between CZ2 and CZ4 (16-34). The grid, with the connection between the busses in each zone and a simplified representation of the complete system are given in figure 3 and 4 . Initially the grid in each of the control zones 


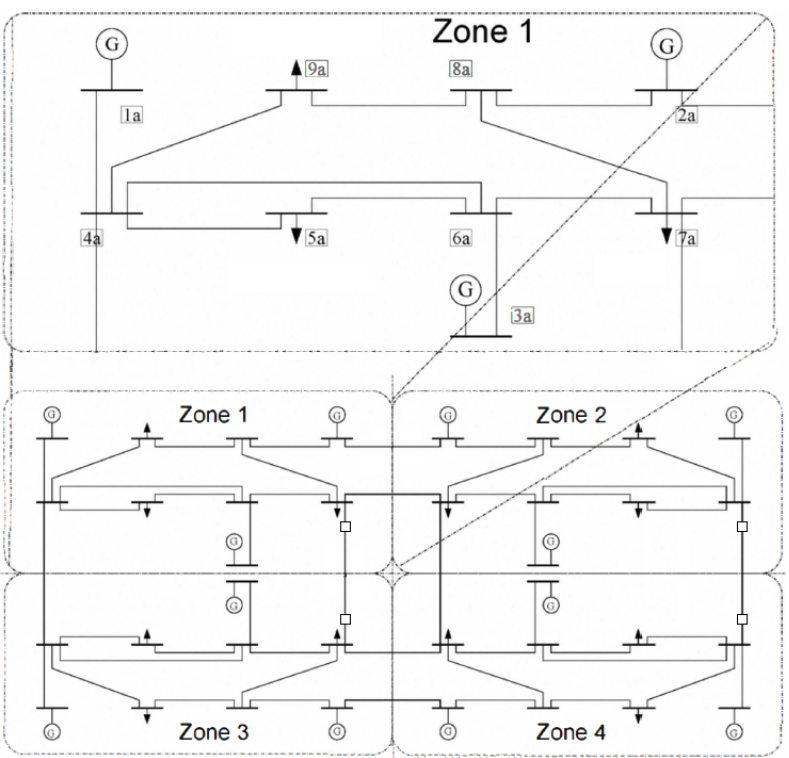

Fig. 4. The cloverfield test system [2]

is considered to be a "copper plate", meaning without internal line limits.

\section{A NEED FOR MORE COORDINATION}

A possible case that could cause a control zone to enter the alert or even the emergency state, can be the tripping of multiple generation units in the same zone during a heavily loaded situation on the system. Such an event could be caused by a natural calamity. The complete synchronous power system still is considered to be $\mathrm{N}-1$ secure, but due to the contingency the affected zone has used all its available reserves. As such this is not a generation problem, but a matter of managing congestions and overloads. During this situation the transfer of power to the zone that lost the generation unit will increase, due to the help automatically delivered by neighbouring control zones. To avoid that the complete power system will collapse, only temporal overloading of lines is allowed during this process. To overcome this event, the TSO, of the control zone that lost a generation unit, will try to start up new generation to return to the $\mathrm{N}-1$ secure state. The critical time frame, thus is the time needed to start up this extra generation. If during this time interval another event occurs in the same control zone, then the system can move over the secure border. As the system of this control zone is not designed to cope with this situation, help from the neighbouring zone is needed to overcome this event.

The increase of the power flow into this zone can cause the violation of thermal limits of lines or cables connecting the different zones or within one zone. The use of power flow controllable devices influences the flow between different zones and can be used to manage the available capacity more efficiently. In this paper HVDC is used to show the influence. The number of installed HVDC connections in Europe has been increasing during the last decade and is going to increase even more in the next ten years [6]. Up till now connection

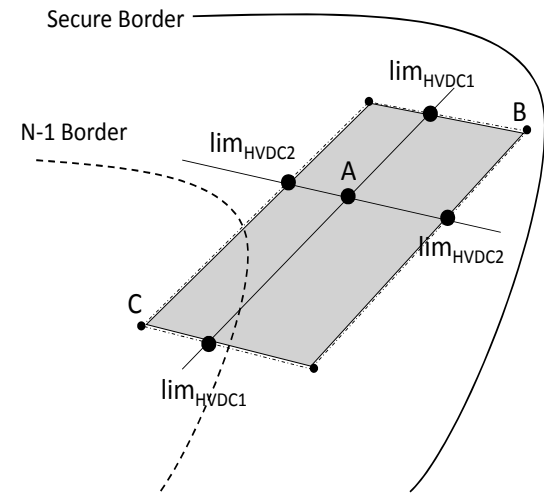

Fig. 5. The domain of the HVDC

have mainly been built between different asynchronous zones such as between continental Europe and the UK, or between continental Europe and Scandinavia. But the number of HVDC connections within a single synchronous zone is also increasing. Examples are the interconnection between France-Spain [7] and the planned connection Belgium-Germany [8].

The set-points of all these HVDC lines have an influence on the operating point of the system. Depending on ownership and operating regime, the set-points of the different PFCs (connecting to the TSOs control zone) are fully, partially or at a cost available to the TSO. PFCs at cross-border connections are generally operated by either one TSO, two TSOs jointly or by an independent operator. TSOs generally have little influence on the set-points of PFC which are located outside their control area. Looking at the grid in figure 3, this means that without coordination it is impossible for the TSO of CZ4 to influence the set-point of HVDC line 13-31, but not the setpoint of the connection 7-25. Thus, a lack of coordination will limit the potential that is inherently available by the HVDC technology. The TSO of the affected control zone, can only to a certain extend influence the power transfer to his zone, which in some cases could lead to the loss of load. If all the HVDC connections, which influence the transfer to this affected zone, would adjust their set-points, a larger amount of support might be given. In such a case, the amount of shedding can be reduced or load shedding might no longer be needed.

In operating point $A$ in figure 5 the system is no longer $\mathrm{N}-1$ secure. The effect of HVDC control on the operation point is shown as a shaded area. Depending on the set-point of the controls, the system can be operated closer to or farther from the security limits. In this case the operation point closest to the security border which is reachable through manipulation of HVDC set-points is point $\mathrm{B}$.

If the set point of HVDC2 is fixed, then the only control variable is the set point of HVDC1. Changing the set point of HVDC1 causes the operation point of the system to move along the axis of HVDC1. Control of the HVDC connection is possible between the two limits (limit ${ }_{H V D C 1}$ ) given in the figure. The magnitude of the limits is determined by the rating of the HVDC line. The influence, of the direction of the flow through the HVDC line, on the position of the operating point 


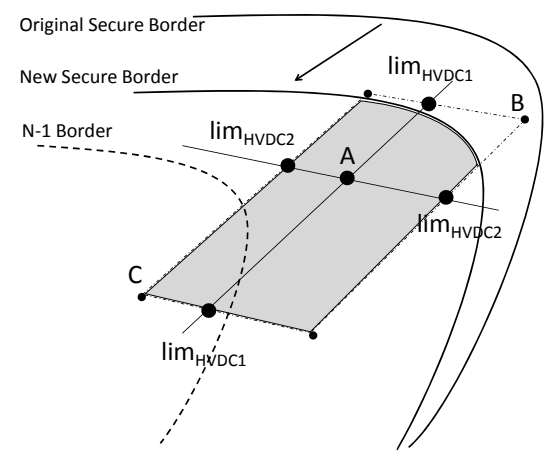

Fig. 6. Reduced operating area

is unclear. Therefore in figure 5 no sign is assigned to these limits. Depending on in which direction the set point changes, the operation point can move to a more secure or less secure point. The operation point is considered to be more or less secure depending on what the impact of a subsequent event on the security of the grid would be. Here, a more secure point is a point situated a larger distance from the secure border. Therefore $\mathrm{C}$ is considered to be more secure than A. The closer the point moves to point $\mathrm{C}$, the larger the margin on the transmission lines. A less secure point is situated closer to the secure border. Therefore B is considered less secure than A. When operating in point $B$ the margin on the capacity of the transmission lines will be smaller than in point $\mathrm{A}$, though larger transit may be possible. The disadvantage of being close to the secure border is that a subsequent event could result in an operating point outside the secure space. Load shedding might still be possible, but dynamic simulation is necessary to prove that it is possible to return to a stable point.

In case that there is no international coordination among TSO's, but only coordination between neighbours the operating point will only be able to move along one of these axis and the influence on the transfer and cost reduction will be limited. In the case that the set point of HVDC line 2 is fixed the only control variable is the set point of HVDC line 1 which can shift the operation point between the two limits of HVDC1. A more coordinated method of determining the set- points of the HVDC connections might increase the transfer between zones, which could lead to a reduction of load shedding during multiple contingency situations. This point is located in the shaded area in figure 5 .

Besides the limits of the controllable devices, also other boundaries conditions exist, that can be more stringent. In figure 6 the thermal limits of the branches within each zone are taken into account. Here it seems that in some situations they are the limiting factor in the control. This is represented by the reduction of the shaded area given in figure 6 . While only two HVDC connections are considered here, the same theory can be extended for multiple HVDC connections.

\section{Formulation OF THE OPF AND COST FUNCTION}

During the simulations an extended version of the standard DC OPF model has been used. MATPOWER has been used

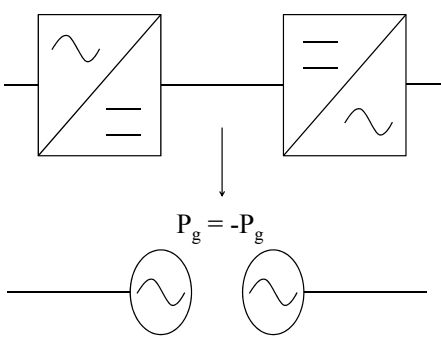

Fig. 7. Model used for HVDC line

to do the simulations [9]. In a DC power flow calculation, the voltage magnitudes are considered to be constant and equal to one, the resistances are neglected and the sin of small angle differences are approximated by the angle differences themselves. The optimisation function is given in equation 1 , which gives the summation of the cost of the active power generation. This formulation also takes into account the cost of load shedding, as load shedding is modeled as a generator producing a negative active power. The optimisation function is subject to the following constraints: load balance in each node (2), line flow limits (3), generation limits(4).

$$
\begin{gathered}
\min \Sigma\left(P_{g} * C_{g}\right) \\
P_{b u s}\left(\theta, V_{m}\right)+P_{d}-P_{g}=0 \\
F_{\text {min }}<F(\theta)<F_{\text {max }} \\
P_{\text {min }}<P_{g}<P_{\text {max }}
\end{gathered}
$$

The HVDC connections have been modeled in the OPF by adding two dummy generators (one to each of the busses the DC link connects to) and an extra constraint, which makes sure that their output should be of the same magnitude but with opposite sign (figure 7). In the OPF calculation the cost can be set to a value which represent the cost of using the HVDC link. In the simulation the cost has been set below the cost of the cheapest generator, therefore the HVDC link is considered as a first solution.

The loadshedding cost of the system has been calculated by taking the summation of the amount of load shed multiplied by the value of lost load. It is important to notice that it is very difficult to give an accurate estimation for the value of lost load, as it is depends on the customer, the time of the loss and the duration [10]. As the load shedding in practice is considered as the ultimate solution, the value of lost load is set to be higher than the cost of the most expensive generator in the system. In this case the value of lost load is set to 400EUR/MWh.

\section{RESUltS}

A serious contingency is considered (e.g. the tripping of two generation units), which causes the system to operate in the region between the N-1 secure and the system wide secure border. The tripping of an extra generation unit, in the same control zone (here CZ4), could cause the operation point to move over the secure border which can lead into a 
collapse of the system. During such a situation, the TSO of this region could ask for more support of his neighbouring TSOs to overcome the initial phase of the event and in the meanwhile start-up extra generation units, to rebalance the generation and load off-take within his own control zone. The tripping of this extra generation unit is here modeled as an increase of the load in this zone. This is done because of two reasons. Firstly, due to the higher penetration of renewable generation units in the distribution grid, a change of weather conditions can cause a sudden change of generation output which is no longer concentrated on one bus. Therefore it is straight forward to model this generation reduction as a load increase, which is distributed over all the busses with load. Secondly this is a convenient manner to increase the crossborder transfer in small steps, to investigate the system limits that are reached. This stepwise decrease of generation could also be modeled by changing the limits of a generator.

TABLE I

MAXIMUM TRANSFER POSSIBLE BEFORE LOADSHEDDING (INSTALLED CROSS BORDER CAPACITY FOR ZONE 4 IS 900MW)

\begin{tabular}{ccccc}
\hline & \multicolumn{2}{c}{ Only crossborder limits } & \multicolumn{2}{c}{ With internal limits } \\
Case & MW & \% of cap & MW & \% of cap \\
\hline F1F2 & 580 & 64.44 & 440 & 48.89 \\
O1F2 & 723 & 80.33 & 552 & 61.34 \\
F1O2 & 728 & 80.89 & 491 & 54.56 \\
O1O2 & 760 & 84.44 & 576 & 64.00 \\
\hline
\end{tabular}

The main results of the simulations are shown in table I. Firstly, a "copper plate model"has been used in the simulations for each of the control zones. In this model the branch limit of the branches within each zone have been set at a very high limit. Therefore the system limits, and thus the secure border, are defined by the limits of the tie-lines between the different control zones and the limits of the HVDC lines. There are four case considered: F1F2, F1O2, O1F2,O1O2. The HVDC line between CZ1 and CZ3 is represented by number 1 and the HVDC line between CZ2 and CZ4 is represented by number 2 . The " $F$ " represents that the set-point of the considered HVDC-line has been kept fixed to the pre-event value. While in case of an "O", there has been coordination between the TSO's connected by the considered HVDC line and the set point has been chosen to minimize the effect of the events. In this case this means that there is a minimisation of the amount of load that is shed. The case F1F2 represents that there would be no communication between TSOs and the set-points of both HVDC-lines are fixed to the pre-event situation. As a result the support by the other regions to the control zone is limited. At a transfer of $580 \mathrm{MW}$ to CZ4, the loadshedding in zone 4 initiates (As all reserve generation of zone 4 is depleted, this increase of load is equal to the amount of power transferred from the other regions to zone 4). The cases $\mathrm{O} 1 \mathrm{~F} 2$ and F1O2 are the cases in which there is only one of the two HVDC lines in the system that changes his set points. The amount of power that can be transferred to zone 4 increases to $723 \mathrm{MW}$ and $728 \mathrm{MW}$ respectively. Of these cases without international coordination, the case $\mathrm{F} 1 \mathrm{O} 2$ is the most probable, as collaboration between neighbouring TSOs is common practice. The TSO of control zone 4 regularly communicates with the TSO of zone 2 and 3 concerning the events on his grid, but not directly with the TSO of control zone 1. Therefore a fast change of the set-point for HVDC 1 is not evident. Though through international coordination, the transfer to zone 4 can be further increased by changing the set-points of both HVDC lines in an optimal way. This is the graph given by $\mathrm{O} 1 \mathrm{O} 2$ increasing the transfer to $760 \mathrm{MW}$. In comparison to the case $\mathrm{F} 1 \mathrm{O} 2$, this is an increase of the use of transfer capacity by $3.55 \%$.

The same simulation can now be made for the case that the branch limits in each zone are taken into account. The results of these simulations are shown in table I. These thermal limits of the branches add extra boundary limits to the secure operating space and can cause a further reduction of this space. In normal security assessments, not all constraints are taken into account to facilitate the computational effort and the analysis of the results. A selection of most stringent constraints, called critical branches, is made. However when large changes in operating condition occur, additional constraints need to be considered. As already shown in the abstract figure 6, this is also visible in the simulation results in table I. Due to the extra limits the maximum transfer to zone 4 , without causing load shedding, is reduced for each of the four cases. The full operating space of the HVDC lines is no longer available. Still it remains clear that cooperation on an international scale can improve the transfer and as such reduce the amount of load that needs to be shed. Here the increase of use of capacity is 9.44\% between the $\mathrm{F} 1 \mathrm{O} 2$ and $\mathrm{O} 1 \mathrm{O} 2$ case.

It should also be noted that coordinated control of the HVDC lines already improves the social welfare before load needs to be shed. This can be noticed by plotting the cost in function of the transfer to zone 4. The cost function is a summation of the generation cost and the cost of loadshedding.

In figure 8 the cost function has been plot for the $\mathrm{F} 1 \mathrm{O} 2$ and the $\mathrm{O} 1 \mathrm{O} 2$ case. At a low transfer to zone four, both curves coincide. As the cost curve here is solely determined by the generation cost, this means that generation costs are equal for both cases. As the transfer flow to zone 4 is further increased

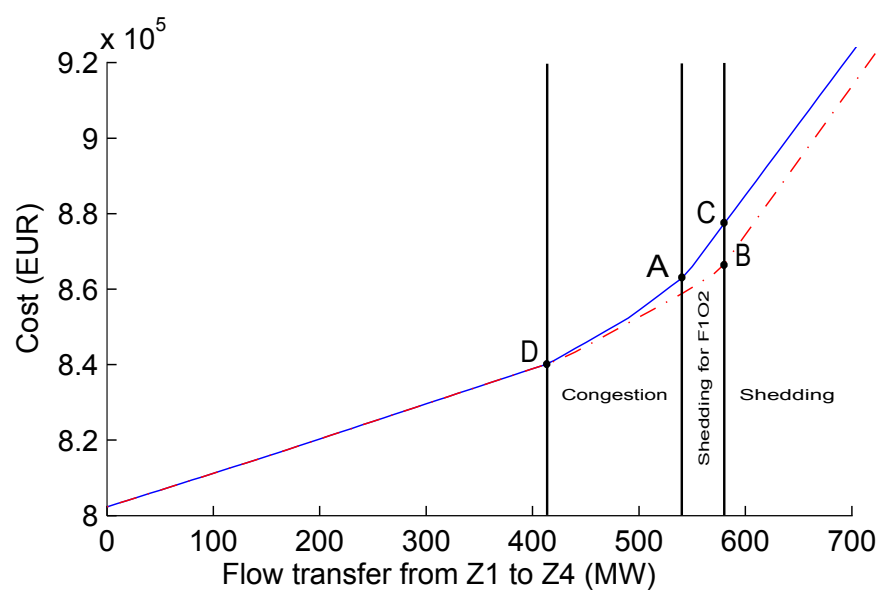

Fig. 8. Cost curve of cases $\mathrm{F} 1 \mathrm{O} 2$ and $\mathrm{O} 1 \mathrm{O} 2$ (internal limits taken into account) 
both curves no longer coincide. The difference between both cost curves is proportional to the cost caused by the congestion of one of the branches in the system. Here it is clear that an interzonal coordination can reduce the generation cost by using the power system in a more optimal way. The point A in the figure 8 gives the starting point of shedding for $\mathrm{F} 1 \mathrm{O} 2$, if there is a need for more transfer to zone 4 . The similar point for the case $\mathrm{O} 1 \mathrm{O} 2$ is given by the point $\mathrm{B}$. Moving from point $\mathrm{A}$ to B there is both a horizontal as a vertical shift. The horizontal shift is caused by a more optimal use of the grid by choosing the set point in an intelligent way. By coordinated control of the HVDC lines in the power system, it is possible to extend the grid capabilities and postponing the shedding of load. The vertical shift A-B is caused by the extra generation needed to supply the load. Though this cost is considerably lower than cost difference B-C. C gives the cost if the same transfer to zone four is applied in the $\mathrm{F} 1 \mathrm{O} 2$ case. In point $\mathrm{C}$ part of the load in the system has been shed. The inclination of the cost curve between point $\mathrm{A}$ and $\mathrm{C}$ is determined by the amount of load that is shed if the transfer to zone four is increased by one MW. The difference B-C is also proportional to the load shed, as the cost for load shedding has been taken the same for all the loads.

\section{Proposed COORdinAtion METHodology}

The proposed methodology for coordinated control of PFCs is depicted in figure 9. To be able to do the necessary simulations the coordinating organization needs to receive grid data and be able to monitor where a contingency is happening on the power system. Based on this information, simulations are done to search for a more optimal set-point for the PFCs. If set-points, which lead to a more secure state of the power system exist, then these will be communicate to the TSOs. If not, the standard emergency procedures as described in policy 5 of the operation handbook are applied [5]. Based on these new set-points, the TSO will need to do extra simulations to check whether all local limits are respected. If some limit is violated, this will again be communicated to the coordinating organization, so they can perform a new simulation. This loop iterates untill acceptable set-points are found. Subsequently, the application of the current emergency procedures will bring the system back to the normal operating state. The tasks of the coordinating organization are given in the box with the dashed line in figure 9. This coordinating organization can be an organization like CORESO or TSC.

\section{CONCLUSION}

In this paper the effect of the active power set points for a system with multiple HVDC lines has been investigated on a small test grid consisting of four control zones interconnected by several tie-lines and two HVDC lines. Each of the zones is considered to be operated by another TSO. It has been shown that it is possible to reduce generation cost and load shedding by extending the grid capabilities through correct set-point selection based on international coordination. Secondly, due to this more optimal use of the grid, the point, at which the load

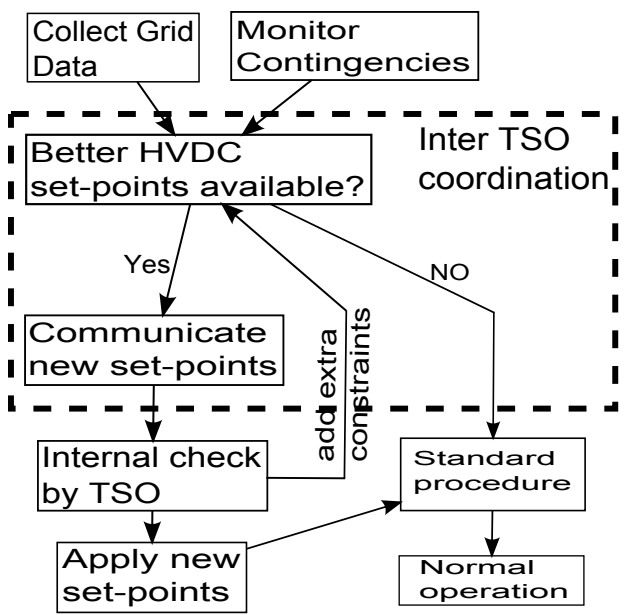

Fig. 9. Proposed coordination methodology

shedding is initiated, can be shifted to a higher power transfer. In this way, it is possible to operate the grid closer to its limits by giving more support to the zone, that has used his reserves. This results in a reduction of the amount of load that needs to be shed or can even avoid any load shedding. Therefore, as it is impossible for one TSO to determine the most optimal setpoints for all the HVDC lines in the system, the need for more international coordination is increasing. Certainly, taking into account the planned increase of the number of HVDC lines in the European system, more coordination is needed to increase the social welfare, by cost reduction, and to operate the system in a more secure way.

\section{REFERENCES}

[1] P. Buijs, D. Bekaert, S. Cole, D. V. Hertem, and R. Belmans, "Transmission investments in europe: Going beyond standard solutions," Energy Policy, vol. 39, pp. 1794-1801, 2011.

[2] D. Van Hertem, R. Eriksson, L. Söder, and M. Ghandhari, "Coordination of multiple power flow controlling devices in transmission systems," in IET ACDC, oct 2010, pp. $1-6$.

[3] D. Van Hertem, J. Rimez, and R. Belmans, "Power flow controlling devices as a smart and independent grid investment for flexible grid operations: Belgian case study," IEEE Trans. Smart Grid, 2013, accepted for publication.

[4] A. Oudalov, R. Cherkaoui, A. Germond, and M. Emery, "Coordinated power flow control by multiple facts devices," in Power Tech Conference Proceedings, 2003 IEEE Bologna, vol. 3, june 2003, p. 5 pp. Vol.3.

[5] ENTSO-E, "Operations handbook policy 5: Emergency operations," ENTSO-E, Tech. Rep., 2012. [Online]. Available: https://www.entsoe.eu/publications/

[6] ENTSO-E, "10-year network development plan 2012," ENTSO-E, Tech. Rep., 2012. [Online]. Available: https://www.entsoe.eu/majorprojects/ten-year-network-development-plan/tyndp-2012/

[7] P. Grollier, "France/spain reinforcement," RTE, Tech. Rep., 2010. [Online]. Available: http://realisegrid.rse-web.it

[8] ENTSO-E, "Regional investment plan north sea," ENTSO-E, Tech Rep., 2012. [Online]. Available: www.entsoe.eu

[9] R. Zimmerman and C. Murillo-Sanchez, "Matpower: Steady-state operations, planning, and analysis tools for power systems research and education," IEEE Trans. on Power Systems, vol. 26, pp. 12-19, 2011.

[10] S. Stoft, Power system economics: Designing Markets for Electricity. John Wiley \& Sons, 2002. 